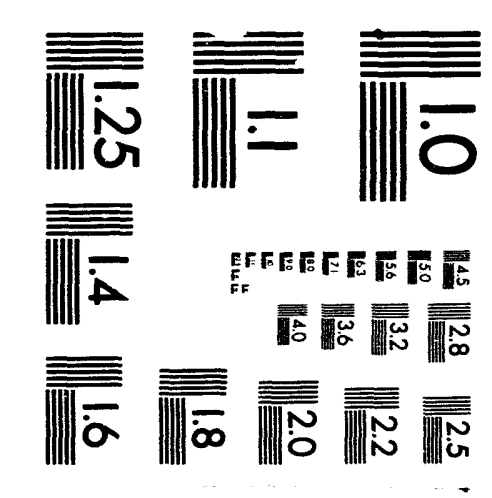





\title{
Conf $-93 / 109--37$ \\ GAS-PHASE SILICON ATOM DENSITIES IN THE CHEMICAL VAPOR DEPOSITION OF SILICON FROM SILANE
}

SANOS3-1489C

\author{
MICHAEL E. COLTRIN, WILLIAM G. BREILAND, AND PAULINE HO
}

Sandia National Laboratories, Albuquerque, NM 87175

\section{ABSTRACT}

Silicon atom number density profiles have been measured using laser-induced fluorescence

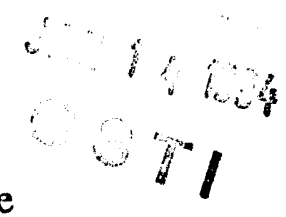
during the chemical vapor deposition of silicon from silane. Measurements were obtained in a rotating-disk reactor as a function of silane partial pressure and the amount of hydrogen added to the carrier gas. Absolute number densities were obtained using an atomic absorption technique. Results were compared with calculated density profiles from a model of the coupled fluid flow, gas-phase and surface chemistry for an infinite-radius rotating disk. An analysis of the reaction mechanism showed that the unimolecular decomposition of $\mathrm{SiH}_{2}$ is not the dominant source of Si atoms. Profile shapes and positions, and all experimental trends are well matched by the calculations. However, the calculated number density is up to 100 times smaller than measured.

\section{INTRODUCTION}

The chemical vapor deposition (CVD) of silicon from silane is the simplest system of use in the microelectronics industry. As such, it is a system often chosen for research into the fundamental mechanisms of CVD. Under the usual conditions for low-pressure CVD (LPCVD), homogeneous decomposition of silane is negligible. However, at higher pressures homogeneous pyrolysis and subsequent gas-phase reactions of intermediate species can occur quite readily.

Studying the fundamental chemistry occurring in a CVD system using a commercial reactor is very difficult. The chemical kinetics is strongly coupled to the gas-phase temperature and velocity fields, which can be quite complicated in commonly used commercial CVD reactors. In addition, modeling detailed chemical kinetics coupled to complex fluid flow is still computationally prohibitive. Several years ago, we presented comparisons between a 2dimensional boundary layer flow model of the silane CVD system [1] and in situ measurements of gas-phase temperatures [2], silane density profiles [2], silicon dimer density profiles [2], and silicon atom density profiles [3] under a wide range of conditions. Generally, good agreement was obtained between model and experiment. However, the idealized 2-D flow in the model was a crude approximation to the actual experimental reactor in which the measurements were performed (which contained various viewing ports). Therefore, when discrepancies occurred between model and experiment it was difficult to be sure that the errors were due to errors in the chemical kinetics portion of the model, or to inaccurate modeling of the fluid flow.

Very simple fluid flow for CVD is found in the rotating disk reactor (RDR). Von Karman showed that the flow above an infinite-radius rotating disk can be reduced to just one mathematical dimension, the distance above the disk. Breiland and Evans [4] presented an analysis and experimental design of a finite-radius RDR which mimics the ideal, 1-D behavior from the von Karman (similarity) solution. Coltrin, Kee, and Evans [5,6] developed a 1-D computer model (SPIN) of the coupled fluid flow, gas-phase and surface chemistry using the rotating-disk similarity solution.

This paper discusses measurement of $\mathrm{Si}$-atom density profiles in a rotating disk reactor and comparisons with calculations using the 1-D model. As has been shown before [4], the fluid flow in this reactor is well-understood and easily modeled; gas-phase temperature profiles match the 1-D solution essentially exactly. The present comparisons of Si-atom profiles provide a well-controlled test of the chemical kinetics portion of the model, with the fluid flow taken as a "given." 


\section{EXPERIMENT}

The experimental RDR has been described elsewhere [4]. The cylindrical reactor is built with double quartz walls ( $-10 \mathrm{~cm}$ i.d.) through which cocling water flows. The rotating disk consists of a 75-mm-diameter stainless steel, resistively heated canister, on which a 51-mmdiameter silicon wafer is placed. The gases $\left(\mathrm{SiH}_{4}, \mathrm{He}\right.$, or $\left.\mathrm{H}_{2}\right)$ enter the reactor through a diffuser screen and exit the cell at the bottom. The flow rate is set to match the amount required by the ideal 1-D flow [4] for a desired operating pressure, rotation rate, and disk temperature. Si atoms are detected using laser-induced fluorescence (LIF), essentially as described elsewhere [3]. The excitation source was the frequency doubled and mixed output near $250 \mathrm{~nm}$ from a pulsed YAG-pumped dye laser. The fluorescence was collected at $90^{\circ}$, spectrally selected with a 0.5 meter monochromator, and detected with a photo multiplier tube. The Si atom density measurements were done by tuning the laser to the $J=2 \rightarrow 1$ line of the $4 s^{3} \mathrm{po} \rightarrow 3 \mathrm{p}^{2}{ }^{3} \mathrm{P}$ transition at $250.7 \mathrm{~nm}$ while monitoring the signal at the $\mathrm{J}=2 \rightarrow 2$ transition at $251.6 \mathrm{~nm}$. Spatial profiles of Si atom densities were obtained by moving the RDR vertically relative to the laser beam and collection optics. A UV absorption measurement was performed to calibrate the LIF signal, and thus obtain absolute number densities. More detail about the experiment will be given elsewhere [7].

\section{MODEL}

The SPIN computer code [6] solves the 1-D equations for the three velocity components, the gas temperature profile, gas-phase mole fractions, surface species site fractions, and deposition rates. The model accounts for homogeneous decomposition of the silane reactant and subsequent reactions of the gas-phase intermediates that are produced. The complete gas-phase silane pyrolysis reaction mechanism used in this study is given in Table 1 . The complex temperature and pressure dependence of many of these reactions was parameterized in a Troe form $[8,9]$. The low-pressure and high-pressure limits of the rate constants are given by

$$
\begin{aligned}
& k_{0}=A_{0} T^{\beta_{0}} \exp \left(-E_{0} / R_{c} T\right), \\
& k_{\infty}=A_{\infty} T^{\beta_{\infty}} \exp \left(-E_{-} / R_{c} T\right) .
\end{aligned}
$$

The rate constant as a function of pressure is then

$$
\begin{gathered}
k=k_{\infty}\left(\frac{P_{r}}{1+P_{r}}\right) F, \\
P_{r}=\frac{k_{0}[M]}{k_{\infty}}
\end{gathered}
$$

and $[M]$ is the total concentration of species in the mixture. In the Troe form, $F$ is given by

The constants are

$$
\log F=\left[1+\left[\frac{\log P_{r}+c}{n-d\left(\log P_{r}+c\right)}\right]^{2}\right]^{-1} \log F_{c e n t} .
$$

$$
\begin{aligned}
& c=-0.4-0.67 \log F_{c e n t} \\
& n=0.75-1.27 \log F_{c e n t} \\
& d=0.14
\end{aligned}
$$

and $F_{c e n t}=(1-a) \exp \left(-T / T^{* * *}\right)+a \exp \left(-T / T^{*}\right)+\exp \left(-T^{* *} / T\right)$. 
The four parameters $a, T^{* * *}, T^{*}$, and $T^{* *}$ are specified as third line of coefficients for reactions $\mathrm{G} 1,2,3,5,6$, and 8 .

The rate expressions for reautions G1 through G7 represent the best summary of silane, disilane, and trisilane kinetics available from the literature [10-12]. Moffat, Jensen, and Carr have performed a non-linear regression to summarize the available kinetic data available for these systems. The rate constants for these reactions were not varied in any of the calculations reported here. In actuality there are uncertainties of factors of 3 or more in each of these rate constants, which lead to potential errors in our calculated Si atom densities. The isomerization reaction $\mathrm{G} 8$ connects the two forms of $\mathrm{Si}_{2} \mathrm{H}_{4}$. There is no reported rate constant for this reaction. Reaction G9 is the main production / destruction route for Si atoms in the mechanism. $\mathrm{P}$ zaction G10 is the main production / destruction route for Si when disilane is used as a starting gas [7], but plays little role in the calculations reported here. If not given explicitly in the table, the reverse rate constants were calculated from the reaction thermochemistry. Thermochemical data from references [5] and [13] were used.

TABLE 1

\begin{tabular}{|c|c|c|c|c|c|c|}
\hline & Reaction & Rate coeffic & nts (see & notes) & & Notes \\
\hline G1 & $\mathrm{SiH}_{4}(+\mathrm{M}) \leftrightarrow \mathrm{SiH}_{2}+\mathrm{H}_{2}(+\mathrm{M})$ & $3.12 \mathrm{E} 09$ & 1.7 & 54710 & & $a, b$ \\
\hline & & $5.214 \mathrm{E} 29$ & -3.545 & 57550 & 2760 & \\
\hline $\mathrm{G} 2$ & $\mathrm{Si}_{2} \mathrm{H}_{6}(+\mathrm{M}) \leftrightarrow \mathrm{H}_{2}+\mathrm{H}_{3} \mathrm{SiSiH}(+\mathrm{M})$ & $\begin{array}{l}-0.4904 \\
9.09 \mathrm{E} 9\end{array}$ & $\begin{array}{l}000.3 \\
1.8\end{array}$ & $\begin{array}{l}209.4 \\
54197\end{array}$ & 2100 & $\mathrm{a}, \mathrm{e}$ \\
\hline & & $\begin{array}{l}1.945 E 44 \\
-0.1224\end{array}$ & $\begin{array}{l}-7.772 \\
793.3\end{array}$ & $\begin{array}{l}59023 \\
2400\end{array}$ & 11.39 & $\begin{array}{l}c \\
d\end{array}$ \\
\hline G3 & $\mathrm{Si}_{2} \mathrm{H}_{6}(+\mathrm{M}) \leftrightarrow \mathrm{SiH}_{4}+\mathrm{SiH}_{2}(+\mathrm{M})$ & $\begin{array}{l}1.81 \mathrm{E} 10 \\
5.09 \mathrm{E} 53\end{array}$ & $\begin{array}{l}1.7 \\
-10.37\end{array}$ & $\begin{array}{l}50203 \\
56034\end{array}$ & & $\begin{array}{l}\mathrm{a}, \mathrm{e} \\
\mathrm{c}\end{array}$ \\
\hline & & 4.375E-5 & 438.5 & 2726 & 438.2 & d \\
\hline G4 & $\mathrm{H}_{2}+\mathrm{H}_{3} \mathrm{SiSiH} \leftrightarrow \mathrm{SiH}_{4}+\mathrm{SiH}_{2}$ & $9.41 \mathrm{E} 13$ & 0 & 4092.3 & & $a, e$ \\
\hline & & $9.43 \mathrm{E} 10$ & 1.1 & 5790.3 & & f \\
\hline G5 & $\mathrm{Si}_{3} \mathrm{H}_{8}(+\mathrm{M}) \leftrightarrow \mathrm{SiH}_{4}+\mathrm{H}_{3} \mathrm{SiSiH}(+\mathrm{M})$ & $3.73 \mathrm{E} 12$ & 1 & 50850 & & $a, g$ \\
\hline & & $\begin{array}{l}4.36 \mathrm{E} 76 \\
0.4157\end{array}$ & $\begin{array}{l}-17.26 \\
365.3\end{array}$ & $\begin{array}{l}59303 \\
3102\end{array}$ & 9.724 & $\begin{array}{l}c \\
d\end{array}$ \\
\hline G6 & $\mathrm{Si}_{3} \mathrm{H}_{8}(+\mathrm{M}) \leftrightarrow \mathrm{SiH}_{2}+\mathrm{Si}_{2} \mathrm{H}_{6}(+\mathrm{M})$ & $\begin{array}{c}6.97 \mathrm{E} 12 \\
1.73 \mathrm{E} 69 \\
-3.47 \mathrm{E}-5\end{array}$ & $\begin{array}{l}1 \\
-15.07 \\
442\end{array}$ & $\begin{array}{l}52677 \\
60491 \\
2412\end{array}$ & 128.3 & $\begin{array}{l}a, g \\
c \\
d\end{array}$ \\
\hline G7 & $\mathrm{SiH}_{4}+\mathrm{H}_{3} \mathrm{SiSiH} \leftrightarrow \mathrm{SiH}_{2}+\mathrm{Si}_{2} \mathrm{H}_{6}$ & $1.73 \mathrm{E} 14$ & 0.4 & 8898.7 & & $a, g$ \\
\hline & & $2.65 \mathrm{E} 15$ & 0.1 & 8473.4 & & f \\
\hline G8 & 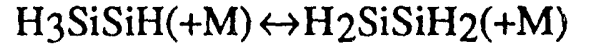 & $2.54 \mathrm{E} 13$ & -0.2 & 5381 & & $\mathrm{a}, \mathrm{h}$ \\
\hline & & $\begin{array}{l}1.099 \mathrm{E} 33 \\
-0.4202\end{array}$ & $\begin{array}{l}-5.765 \\
214.5\end{array}$ & $\begin{array}{l}9152 \\
103\end{array}$ & 136.3 & $\begin{array}{l}c \\
d\end{array}$ \\
\hline G9 & $\mathrm{SiH}_{4}+\mathrm{Si} \leftrightarrow \mathrm{H}_{3} \mathrm{SiSiH}$ & $1.0 \mathrm{E} 12$ & 0 & 5000 & & $\mathrm{a}, \mathrm{h}$ \\
\hline G10 & $\mathrm{Si}_{2} \mathrm{H}_{6}+\mathrm{Si} \leftrightarrow \mathrm{H}_{3} \mathrm{SiSiH}+\mathrm{SiH}_{2}$ & 1.3E15 & 0 & 12600 & & $a, h$ \\
\hline
\end{tabular}

Notes:

a Arrhenius coefficients for the high-pressure limit: $A_{\infty}$ (units depend of reaction order, but are given in terms of moles, sec, and $\mathrm{cm}^{3}$ ), $\beta_{\infty}$ (unitless), $E_{\infty}$ (cal/mole)

b Fits to data in Ref. 10

c Arrhenius coefficients for the low-pressure limit: $A_{0}$ (units depend of reaction order, but are given in terms of moles, sec, and $\mathrm{cm}^{3}$ ), $\beta_{0}$ (unitless), $E_{0}$ (cal/mole)

d Troe parameters (see text): $a, T^{* * *}, T^{*}$, and $T^{* *}$

e Fits to data in Ref. 12

$f$ Arrhenius coefficients for the reverse reaction (units as in note a, above)

$g$ Fits to data in Ref. 13

h Estimated, this work 
Our earlier modeling studies of silane CVD [1,5] included many more reactions and chemical species. In particular the reaction

$$
\mathrm{SiH}_{2} \leftrightarrow \mathrm{Si}+\mathrm{H}_{2}
$$

was included, and it was the major production route for Si atoms in the gas phase. A primary result of this paper is that the reaction of $\mathrm{SiH}_{2}$ cannot be an important route for Si production. It has also been found that species and reactions containing an odd number of hydrogen atoms play little role in the thermal decomposition, and they were thus eliminated from the mechanism. (This, however, would definitely not be the case for plasma-enhanced CVD.) The reaction mechanism has been simplified as much as possible to identify the main pathways for Si atom creation and destruction.

The DAH mechanism [14] for heterogeneous reaction of silane at the deposition surface is used in the model. It consists of a two-site dissociative adsorption of silane, followed by first-order elimination of $\mathrm{H}_{2}$ to the gas [15]. Disilane and trisilane were assumed to react at a rate 10 times faster than the silane rate in [14]. The species $\mathrm{SiH}_{2}, \mathrm{Si}_{2} \mathrm{H}_{2} \mathrm{SiSiH}_{2}$, and $\mathrm{H}_{3} \mathrm{SiSiH}$ were assumed to react with the surface with unit probability.

Figure 1 shows the rate of production (and destruction) of Si due to the reverse of reaction G9 as a function of height above the disk. The peak production rate occurs at roughly $1 \mathrm{~mm}$ above the disk. The production drops as the reactive precursor species $\mathrm{H}_{3} \mathrm{SiSiH}$ is destroyed at the surface. The production rate drops off with increasing distance from the surface as the gas temperature drops. The $\mathrm{Si}$ atoms that are produced diffuse both toward and away from the disk. Further from the disk, Si encounters higher concentrations of unreacted silane, and $\mathrm{Si}$ is destroyed via reaction G9 (in the forward direction). Thus the same gas-phase reaction is both the dominant production and destruction route for $\mathrm{Si}$ in this model.

\section{COMPARISONS BETWEEN MODEL AND EXPERIMENT}

Figure 2 compares measured and calculated $\mathrm{Si}$ atom density profiles for a baseline set of conditions (200 Torr total pressure, 0.33 Torr silane in He carrier, $450 \mathrm{rpm}, 650^{\circ} \mathrm{C}$ disk temperature). It is significant that the measured $\mathrm{Si}$ density profiles have a maximum in the gas phase. This clearly shows that they must be produced via a gas-phase reaction; if they were somehow formed on the surface and then diffused into the gas, the density profile would have its maximum at zero height.

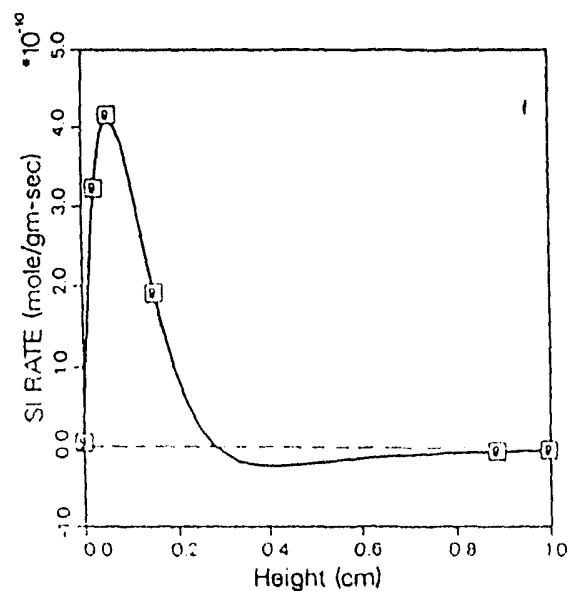

Fig. 1 Production / destruction rate of $\mathrm{Si}$ atoms due to reaction $\mathrm{G} 9$ as a function of height above the disk.

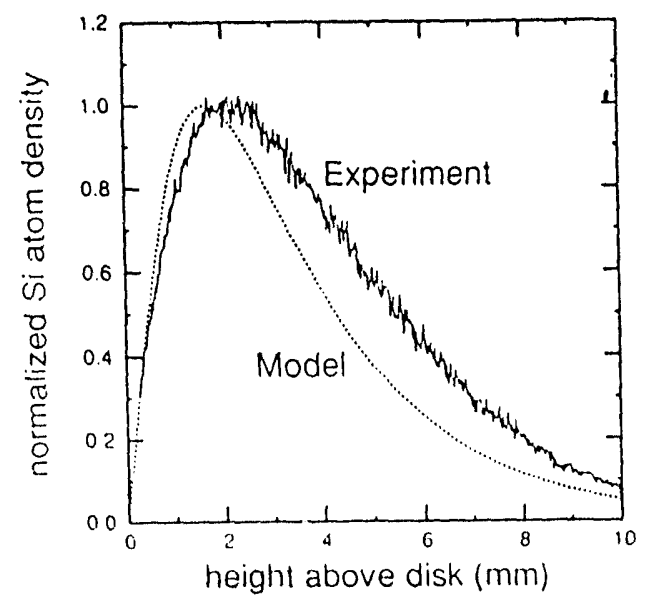

Fig. 2 Measured and calculated Si density profiles. 
The shape of the Si density profile calculated by the model agrees well with experiment. The model matches the position of the maximum in the profile, very near the disk (approximately 2 $\mathrm{mm}$ ). The widths of the profiles also agree well. In the model, the Si density drops at the surface because its production rate goes down (Fig. 1), but also because $\mathrm{Si}$ is destroyed by facile reaction with the silicon surface (unit probability). The drop in Si density further from the surface is also explained by the decrease in production rates in Fig. 1.

The absolute number density measured at the peak of the profile is $2 \times 10^{8} / \mathrm{cc}$ for the baseline conditions. The Si density calculated by the model is $2.1 \times 10^{6} / \mathrm{cc}$, almost exactly 100 times smaller. A number of factors, discussed later, are sources of error in both the experimental and calculated number densities. However, it appears that the calculated number density cannot be raised much by simply increasing the rate constant for G9 (see below).

Addition of $\mathrm{H}_{2}$ to the He carrier decreases the measured LIF signal (and thus the density). Figure 3 shows that the maximum $\mathrm{Si}$ density measured decreases by about $85 \%$ upon addition of 10 Torr hydrogen. The model reproduces the suppression of Si by hydrogen. The experimental and calculated results in Fig. 3 have been normalized at the baseline condition (no $\mathrm{H}_{2}$ ) for ease of comparing the hydrogen effects. The model indicates that the addition of $\mathrm{H}_{2}$ slows the initial silane decomposition by increasing the reverse of G1.

Figure 4 shows that addition of $\mathrm{H}_{2}$ has virtually no effect on the measured shapes of the $\mathrm{Si}$ density profiles. The profiles in Figure 4 have been scaled to the same height, in order to compare the shapes more easily (the scaling of the curves is obtainable from Fig. 3). The Si atom density profiles calculated by the model are shown in Fig. 5. The curves in Fig. 5 have also been scaled to highlight the shapes more clearly. The calculated density profiles using the mechanism presented in Table 1 are also very insensitive to the addition of $\mathrm{H}_{2}$ to the carrier.

The insensitivity of the Si density profiles with respect to added hydrogen was a key experimental finding. It puts very severe constraints on the possible mechanism for $\mathrm{Si}$ production. In fact it is the lack of a "hydrogen effect" that virtually rules out the unimolecular $\mathrm{SiH}_{2}$ reaction as the primary production route, as discussed next.

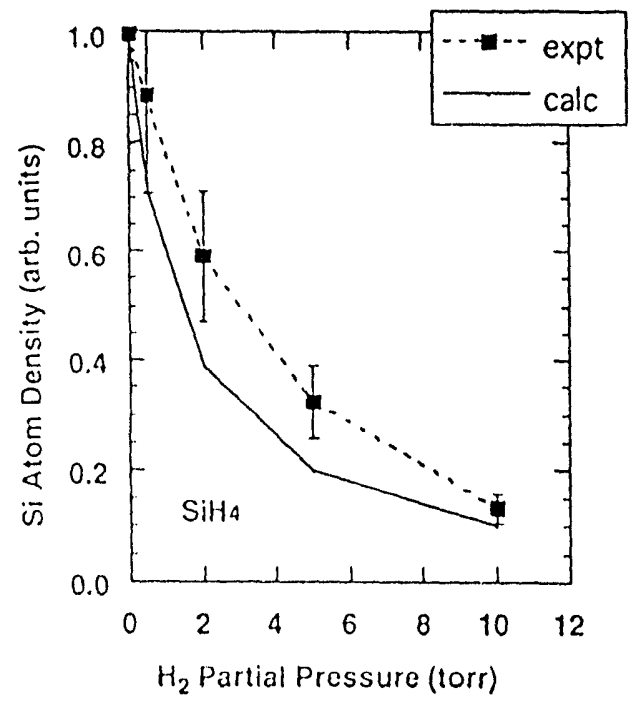

Fig. 3 The effect on the peak Si density of adding $\mathrm{H}_{2}$ to the He carrier gas.

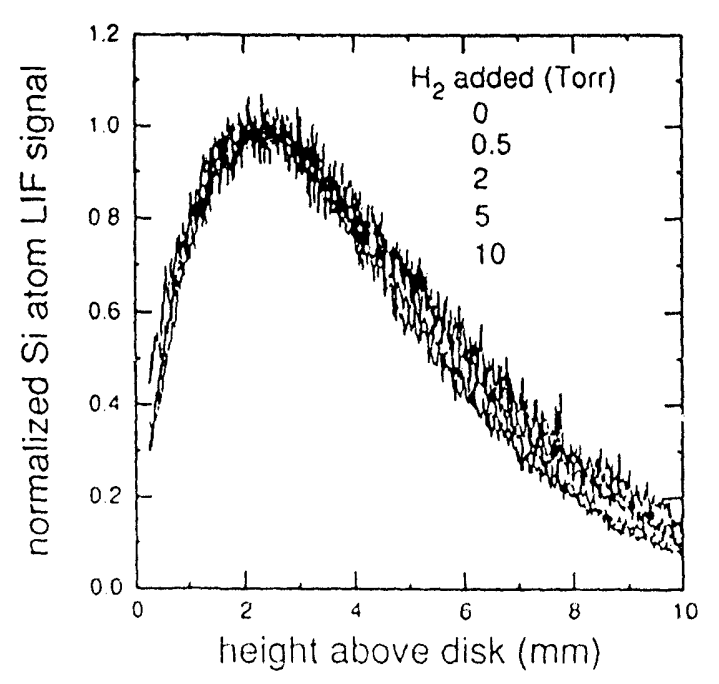

Fig. 4 Shapes of the measured Si density profiles upon addition of $\mathrm{H}_{2}$. Data has been normalized to the same peak height. 


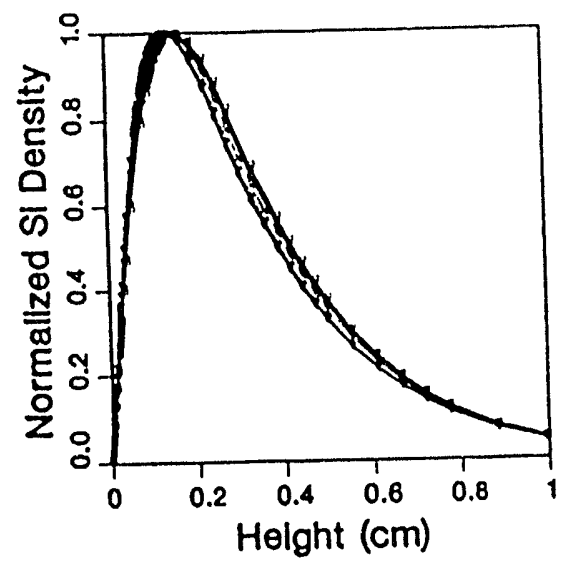

Fig. 5 Shapes of the calculated Si density profiles upon addition of $\mathrm{H}_{2}$. (Curves are normalized. Amounts of added $\mathrm{H}_{2}$ same as in Fig. 4)

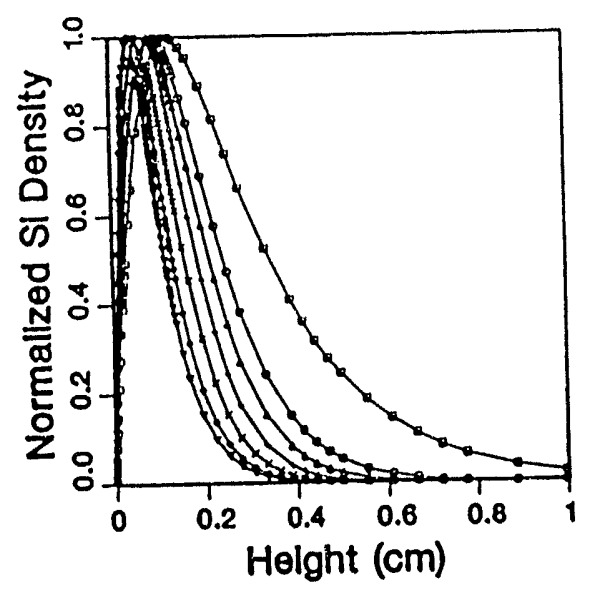

Fig. 6 Shapes of the calculated Si density profiles upon addition of $\mathrm{H}_{2}$ when $\mathrm{SiH}_{2}$ reaction $\mathrm{G11}$-produces gas-phase $\mathrm{Si}$.

The effect on the calculated $\mathrm{H}_{2}$-dependence of adding the reaction (which we will call G11)

$$
\mathrm{SiH}_{2} \leftrightarrow \mathrm{Si}+\mathrm{H}_{2}
$$

to the gas-phase mechanism is shown in Figure 6. A pre-exponential constant of $2 \times 10^{11}$ and an activation energy of $42600 \mathrm{cal} / \mathrm{mol}$ was used for this calculation. (We define this set of reactions and rate constants as Case 2 , and the nominal mechanism in Table 1 as Case 1 ). The peak $\mathrm{Si}$ density for the baseline conditions when $\mathrm{G} 11$ is included is $1.2 \times 10^{8}$, much closer to the experimental value. However, the calculated $\mathrm{Si}$ density profiles narrow significantly as $\mathrm{H}_{2}$ is added to the He carrier. (The broadest profile in Fig. 6 is for the baseline case; the narrowest is for 10 Torr $\mathrm{H}_{2}$ added, with the intermediate curves corresponding to the amounts listed in Fig. 4). The profiles become narrower because, as the amount of hydrogen in the gas increases, the reverse of $\mathrm{G} 11$ becomes more important in destroying $\mathrm{Si}$ atoms. That is, the Si atoms are produced close to the surface and diffuse away. The higher the hydrogen concentration, the shorter the distance before a $\mathrm{Si}$ atom collides with an $\mathrm{H}_{2}$ and is destroyed, thus the narrower profile.

Generally speaking, the rate of the forward direction of $\mathrm{G} 11$ is independent of added $\mathrm{H}_{2}$, but as [H2] gets larger, the more quickly the $\mathrm{Si}$ atoms that are produced will collide with hydrogen and be destroyed by the back reaction. This will be a fundamental behavior of reaction G11; the behavior depends on the equilibrium constant for G11 (ratio of forward to reverse rate constants) no matter what the value of the forward rate constant $k_{f}$ itself. The equilibrium constant, in turn, depends on the reaction thermochemistry, which is pretty well established for these species. For a given $k_{f}$, the reverse rate constant would have to be decreased by roughly a factor of 100 for the narrowing in Si density profile with additional hydrogen to go away. This factor of 100 would require that $\Delta G$ for the reaction be in error by roughly $8500 \mathrm{cal} / \mathrm{mol}$ (at $650^{\circ} \mathrm{C}$ ). The estimated error in $\Delta H$ is less than $1000 \mathrm{cal} / \mathrm{mol}$. It would require an error of about 9 entropy units for $k_{r}$ to be in error by a factor of 100 , which is also too large.

Due to the marked $\mathrm{H}_{2}$-effect on profile widths, reaction $\mathrm{G} 11$ could only be the dominant $\mathrm{Si}$ atom production route if there is some other reaction that can compete with the reverse of G11 for $\mathrm{Si}$-atom destruction. Because the $\mathrm{H}_{2}$ concentration is so great at 10 Torr (relative to most other species in the gas), the only plausible collision partner with a concentration large enough to compete with $\mathrm{H}_{2}$ is $\mathrm{SiH}_{4}$, i.c., in reaction $\mathrm{G} 9$. To test this possibility, a mechanism including 


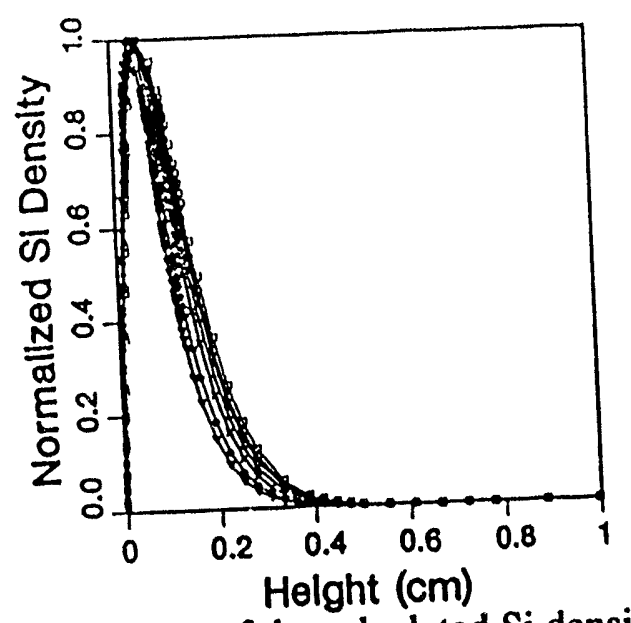

Fig. 7 Shapes of the calculated Si density profiles upon addition of $\mathrm{H}_{2}$ when $\mathrm{SiH}_{2}$ reaction $\mathrm{G} 11$ produces gas-phase $\mathrm{Si}$ and $\mathrm{G} 9$ is increased by a factor of 50 . (Case 3)

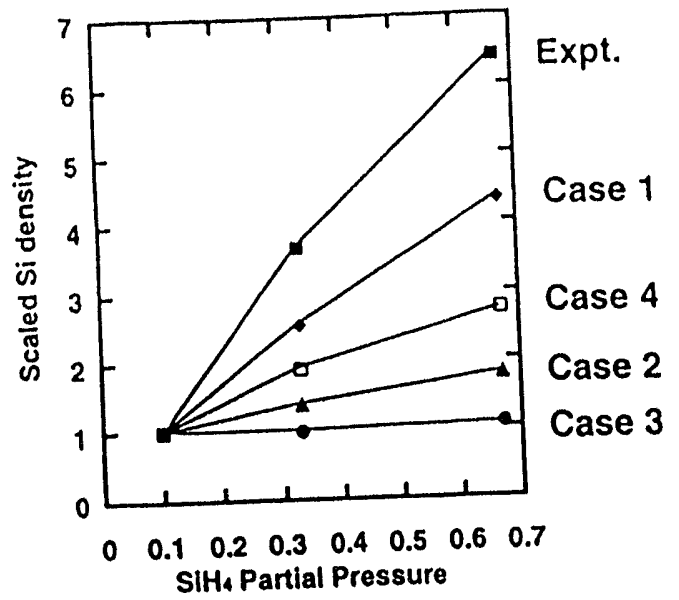

Fig. 8 Dependence of calculated Si density on inlet silane partial pressure. See text for explanation of curves.

Si production by $\mathrm{G} 11$ and increasing the rate for $\mathrm{G} 9$ by a factor of 50 was used to test the effect of $\mathrm{H}_{2}$ on the predicted density profile shapes. (We will refer to this combination of reactions and rate constants as Case 3). The results are plotted in Fig. 7.

Figure 7 shows that the calculated Si density profiles shapes become insensitive to added hydrogen when reaction $\mathrm{G} 9$ is increased to become the dominant Si-destruction route. the profile widths themselves become too narrow, about a factor of three less than

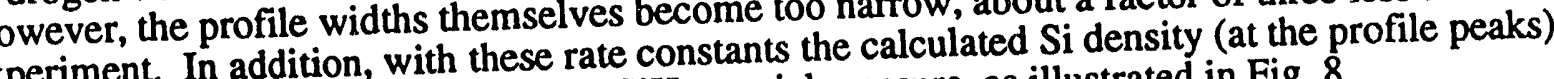
becomes virtually independent of inlet $\mathrm{SiH}_{4}$ partial pressure, as illustrated in Fig. 8.

An almost linear increase in Si-atom density with increasing inlet $\mathrm{SiH}_{4}$ is seen experimentally, as given in Fig. 8. For the combination of rate constants in Case 3, immediately above, there is essentially no increase in the calculated $\mathrm{Si}$ density with increasing silane. For this case, the amount of the precursor $\mathrm{SiH}_{2}$ increases strongly with silane partial pressure, and so the $\mathrm{Si}$ production rate from G11 does, too. However, the rate of destruction of Si also goes up roughly linearly with increasing silane due to reaction G9. The net result is an extremely weak dependence on silane partial pressure. The results calculated for Case 2 (Table 1 mechanism plus reaction G11) also exhibit a very weak dependence of Si density upon inlet silane.

The combination of results just presented essentially rule out reaction G11 as the important producer of Si atoms. Namely, the dramatic narrowing of the Si density profiles with addition of $\mathrm{H}_{2}$ to the carrier seen in Fig. 6, when experimentally the shapes do not change (Fig. 4). The thermochemistry is known too well to allow the needed decrease of the reverse rate constant for reaction $\mathrm{G} 11$ by the necessary factor of 100 . When a fast reaction with $\mathrm{SiH}_{4}$ is allowed to compete with the $\mathrm{H}_{2}$ for the destruction of $\mathrm{Si}$, the profiles become too narrow (Fig. 7) and the Si density becomes independent of silane partial pressure (Fig. 8).

The dependence on silane partial pressure for the nominal mechanism in Table 1 (labeled Case 1 in Fig. 8) shows a slight sub-linear behavior. Earlier in the paper, it was mentioned that the calculated Si density could not be increased by arbitrarily increasing rate constants in the mechanism. This is illustrated by the curve labeled Case 4 in Fig. 8. This calculation starts with the mechanism just as given in Table 1, but increases the rate of G9 by a factor of 10 . With this change, the calculated Si density increases by a factor of 3.5 , but at the expense of further deviation from the nearly linear dependence on silane partial pressure seen experimentally. 
The absolute number density calculated with the nominal mechanism is roughly 100 times lower than measured experimentally. There are number of contributing sources of error, the largest of which will be summarized here. A very high activation energy for Si production was observed, about $135 \mathrm{kcal} / \mathrm{mol}$ [7]. This high activation energy coupled with an uncertainty in our measured surface temperature of about $15^{\circ}$ leads to a factor of 3 uncertainty in the $\mathrm{Si}$ density. The estimated uncertainty in the UV absorption calibration of the absolute number density is a factor of 2 . In the model, we know that the rate constant for the initial silane decomposition is uncertain to within about a factor of 3 . In all of the calculations presented here, the rate constants for reactions G1-8 were held fixed. But, for example, if the rate constant for $\mathrm{Gl}$ is increased by a factor of 3 , the calculated Si density increases by almost exactly the same factor of 3 . Each of the other rate constants in the mechanism are also subject to similar uncertainties in their values. With the combination of uncertainties from these sources, the calculated and experimental Si densities are nearly within their joint error ranges. Still, the large discrepancy is not satisfying.

\section{CONCLUSION}

The controlled environment of the rotating-disk reactor allowed careful tests of the chemical mechanism for creation of gas-phase Si atoms during CVD from silane. A 10-step reaction mechanism was presented which does a good job in matching experimental density profile shapes, the suppression of Si by hydrogen with the profile shapes unchanged, and the dependence upon silane partial pressure. An important conclusion of this work is that the unimolecular decomposition of $\mathrm{SiH}_{2}$ is not the primary route to $\mathrm{Si}$-atom formation.

\section{ACKNOWLEDGMENTS}

We thank Harry Moffat for providing Chemkin fits to the Troe parameterizations, Robert Kee for collaboration in development of the software used here, Greg Evans for consultations on the rotating disk design, Michael Youngman for technical assistance in the design and construction of the rotating disk, and Fran Rupley for programming assistance. This work was performed at Sandia National Laboratories and supported by the U.S. Department of Energy under contract number DE-AC04-94AL85000.

\section{REFERENCES}

1. M. E. Coltrin, R. J. Kee, and J. A. Miller, J. Electrochem. Soc., 131, 425 (1984).

2. W. G. Breiland, M. E. Coltrin, and P. Ho, J. Appl. Phys. 59, 3267 (1986).

3. W. G. Breiland, P. Ho, and M. E. Coltrin, J. Appl. Phys. 60, 1505 (1986).

4. W. G. Breiland and G. Evans, J. Electrochem. Soc. 138, 1806 (1991).

5. M. E. Coltrin, R. J. Kee, and G. Evans, J. Electrochem. Soc., 136, 819 (1989).

6. M. E. Coltrin, R. J. Kee, G. H. Evans, E. Meeks, F. M. Rupley, and J. F. Grcar, Sandia

National Laboratories Report, SAND91-8003, 1991.

7. P. Ho, M. E. Coltrin, and W. G. Breiland (in preparation).

8. R. G. Gilbert, K. Luther, and J. Troe, Ber. Bunsenges. Phys. Chem., 87, 161 (1983).

9. W. C. Gardiner and J. Troe, in Combustion Chemistry, edited by W. C. Gardiner (Springer, Berlin, 1984).

10. H. K. Moffat, K. F. Jensen, and R. W. Carr, J. Phys. Chem., 94, 145 (1991).

11. H. K. Moffat, K. F. Jensen, and R. W. Carr, J. Phys. Chem., 96, 7695 (1992).

12. H. K. Moffat, K. F. Jensen, and R. W. Carr, J. Phys. Chem., 96, 7683 (1992).

13. M. E. Coltrin, R. J. Kec, and J. A. Miller, J. Electrochem. Soc., 133, 1206 (1986).

14. W. G. Houf, J. F. Grcar, and W. G. Breiland, Mat. Sci. and Eng., B17, 163 (1993).

15. K. Sinniah, M. G. Sherman, L. B. Lewis, W. H. Weinberg, J. T. Yates, Jr., and K. C. Janda, J. Chem. Phys., 92, 57(0) (1990).

\section{DISCLAIMER}

This report was prepared as an account of work sponsored by an agency of the United States Government. Neither the United States Government nor any agency thereof, nor any of their employees, makes any warranty, express or implied, or assumes any legal liability or responsibility for the accuracy, completeness, or usefulness of any information, apparatus, product, or process disclosed, or represents that its use would not infringe privately owned rights. Reference herein to any specific commercial product, process, or service by trade name, trademark, mendation, or favoring by does not necessarily constitute or imply its endorsement, recommendation, or favoring by the United States Government or any agency thereof. The views and opinions of authors expressed herein do not necessarily state or reflect those of the 


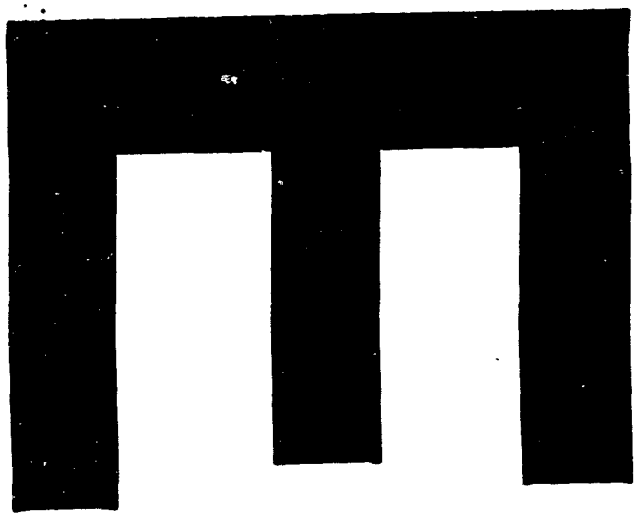

عا
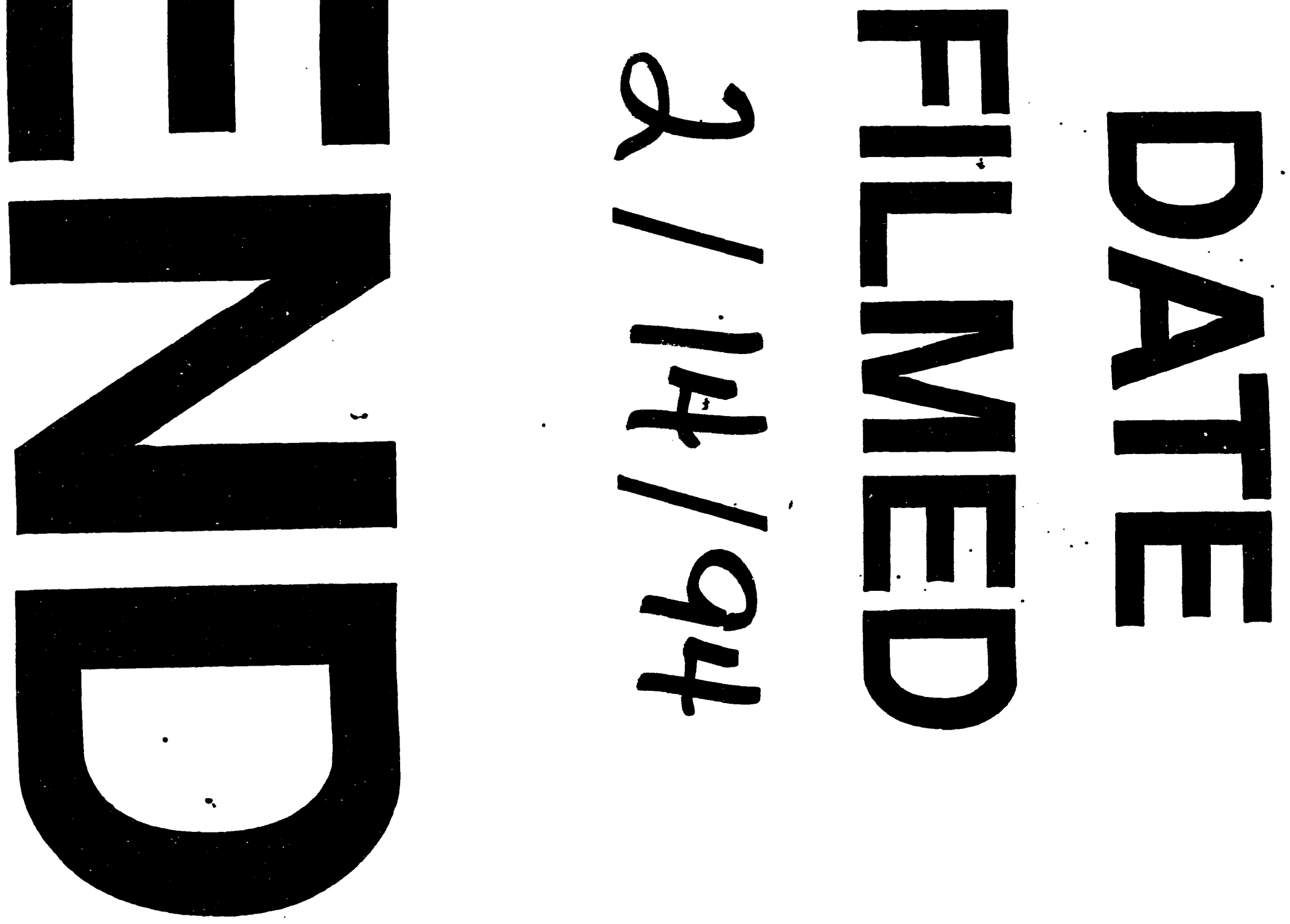
